\title{
Criminologie
}

\section{La criminalité au Canada et aux États-Unis : tendances et explications comparatives (1964-1978)}

\section{Irvin Waller}

Volume 14, numéro 1, 1981

Criminalité et réalités sociales : Québec, Canada, États-Unis, Japon

URI : https://id.erudit.org/iderudit/017131ar

DOI : https://doi.org/10.7202/017131ar

Aller au sommaire du numéro

Éditeur(s)

Les Presses de l'Université de Montréal

ISSN

0316-0041 (imprimé)

1492-1367 (numérique)

Découvrir la revue

Citer cet article

Waller, I. (1981). La criminalité au Canada et aux États-Unis : tendances et explications comparatives (1964-1978). Criminologie, 14(1), 51-84.

https://doi.org/10.7202/017131ar d'utilisation que vous pouvez consulter en ligne.

https://apropos.erudit.org/fr/usagers/politique-dutilisation/ 


\section{LA CRIMINALITÉ AU CANADA ET AUX ETATS-UNIS : TENDANCES ET EXPLICATIONS COMPARATIVES}

Irwin Waller

Au cours des quinze dernières années, aux Etats-Unis comme au Canada, la criminalité et les coûts de la justice pénale ont augmenté de façon continue. Les types de criminalité demeurent cependant d'un niveau inégal et cela est vrai depuis la Deuxième Guerre mondiale et même avant. C'est ainsi que le taux par habitant de crimes de violence, tels que le meurtre, le viol ou le vol qualifié, a plus que doublé. Les taux équivalents ayant trait au vol des biens des individus, des sociétés ou de l'État ont connu une hausse encore plus accentuée. Ces crimes sont dix fois plus fréquents que ceux de violence et forment en Amérique du Nord la base de la criminalité intentionnelle qui menace la sécurité du citoyen ${ }^{1}$. La criminalité reliée à l'alcool, aux drogues, aux véhicules automobiles ou aux affaires a aussi augmente dramatiquement et caractérise la vie quotidienne des deux pays.

Il n'en reste pas moins qu'au Canada, comme aux EtatsUnis, on ne réalise pas que cet essor du crime entraîne un accroissement des pertes financières, des torts physiques et des traumatismes d'ordre émotionnel ${ }^{2}$. Les victimes de crime continuent à être ignorées et ne reçoivent toujours pas d'aide efficace. Au cours des années 70 cependant le public a été sensibilisé par les mouvements féministes aux problèmes des victimes de viol et de violence dans la famille. Parallèlement, les pouvoirs publics ont entrepris aux États-Unis des études de victimisation visant a mesurer ses effets. On a constaté, en outre, une aggravation du malaise qu'éprouve l'opinion publique face à la criminalité, ainsi qu'une détérioration des sentiments de sécurité. Des sondages d'opinion effectués dans les deux pays semblent démontrer que le public veut imposer des peines de plus en plus sévères, tandis

1. Voir pour détails : Quelques tendances de la justice penale canadienne ", Solliciteur général du Canada, 1979a.

2. Pour les quelques victimes dont l'assaillant a éte identifí, s'est posé généralement le problème de restitution des biens volés. Les concernés ont connu des retards judiciaires, des attaques personnelles dans les salles d'audience, et n'ont obtenu en fin de compte que peu de sympathie ou de dédommagement véritables. 
qu'un pourcentage croissant des citoyens évitent certains quartiers des grandes villes, par crainte de victimisation.

Au cours de la même période, les budgets gouvernementaux destinés à la justice pénale ont augmenté plus rapidement que ceux de la plupart des secteurs puisque fort souvent ils ont plus que doublé même en tenant compte de l'inflation. On a assisté ainsi à l'accroissement très prononcé du nombre d'agents de police et de ceux de l'appareil correctionnel et on a construit des prisons à un rythme inégalé jusqu'à présent. Cependant, le nombre de personnes incarcérées n'a pas connu une progression aussi spectaculaire et le taux par 100000 habitants se situe toujours aux environs de 100 pour le Canada et de 200 pour les ÉtatsUnis '. A l'opposé, la proportion des prévenus incarcérés a augmenté et en 1980, par cent mille babitants, le Canada et les Etats-Unis avaient respectivement 15 et 30 personnes qui étaient - présumées innocentes s mais détenues en attendant leur procès 4.

Nous allons donc essayer d'examiner ici ces tendances d'une façon plus détaillée et discuter des explications possibles quant aux taux de croissance et aux différences entre les deux pays. Malheureusement, malgré l'importance de la littérature criminologique, les comparaisons systématiques entre le Canada et les Etats-Unis sont rares. Nous entrons done dans un domaine où on peut appuyer ou mettre en doute plusieurs de nos perceptions et de nos hypothèses relatives à la criminalité traditionnelle. Notre tentative de comparaison n'est, en fait, que préliminaire, mais elle vise à stimuler des études plus approfondies, orientées vers une action plus efficace face à la criminalité et à la poursuite dans les deux pays, d'une plus grande justice sociale.

3. Quant au nombre de personnes détenues la nuit dans les locaux cellulaires de la police - l'endroit carcéral où traditionnellement il y a le plus de chances de suicide - il reste encore fort imprécis.

4. En 1972, il y avait aux Etats-Unis plus de 43000 et au Canada plus de 2400 personnes incarcérées avant leur procès dans les prisons pour adultes (voir Goldkamp 978 p. 7, N.C.J.I.S.S. $1979 \mathrm{c}$, \&uelques tendances en justice pénale canadienne ;, 1979, p. 18, Waller et Chan, 1974). Beaucoup de ces personnes sont libérées dans un délai d'un ou deux jours et donc jugés admíssibles à une mise en liberté dans la collectivité; environ la moitié de ces prévenus ne sont pas condamnés à l'emprisonnement. 


\section{TENDANCES DE LA CRIMINALITE}

\section{Violence}

Analysons tout d'abord la période do 1965 à 1978 pour certaines des tendances de la criminalité de violence 5 . Le graphique I a été élaboré de façon à illustrer à l'aide d'un indice la croissance des crimes de violence :. Pour les États-Unis, cet

\section{GRAPHIQUE I}

Index comparatif des infractions avec violence déclarées par la police par 100000 habitants

Canade - Etats-Unis (1964-1978)

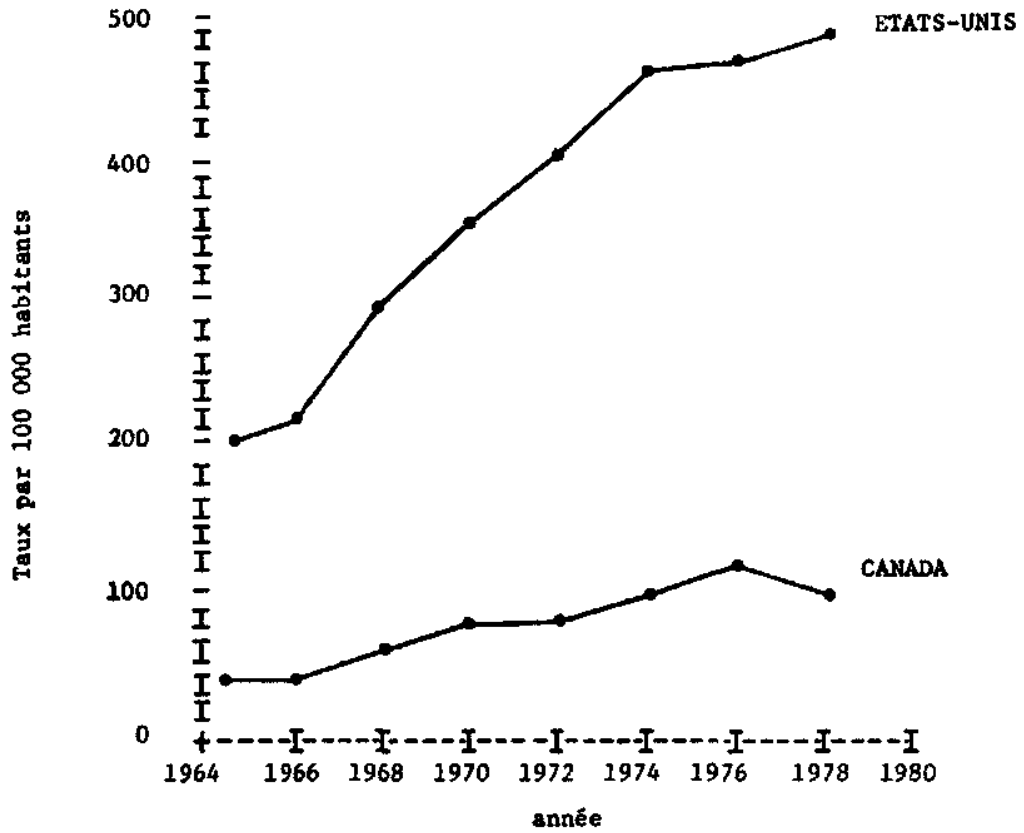

5. Les taux seront calcules dans cet article par 100000 habitants. La répartition des populations des Etats-Unis et du Canada selon l'áge et le sexe n'est pas suffisamment différente pour justifier un taux plus complexe. Les taux de criminalite selon le nombre de cibles possibles ou de criminels en latence rendraient la lecturo difficile ou auraient pour résultat d'embrouiller le lecteur puisque de toute façon, ils sont difficiles à définir de manière satisfaisante.

6. Les graphiques I à III ont eté préparés par la division de la statistique du ministère du Solliciteur général du Canada a partir des Statistiques Canada (1965-1971), (1972-1978) et FBI Uniform Crime Reports (19641978). 
indice a été basé sur les délits tels que le vol qualifie, les voies de fait graves, le viol et le meurtre. Au Canada, il a été établi sur la base du vol qualifié, des coups et blessures, de la tentative de meurtre, du viol, du meurtre et de l'homicide involontaire coupable, puisqu'il fallait tenir compte des différences des définitions légales et des compilations statistiques qui en découlent ?.

Comme on le constate a la lecture du graphique ci-dessus le taux des crimes de violence par rapport a la population a augmenté plus vite aux États-Unis qu'au Canada où d'une manière générale il demeure largement inférieur.

Un indice du crime de violence comme celui-ci varie, en outre, de façon importante à l'intérieur des deux pays concernés. C'est ainsi qu'aux États-Unis les taux de crimes de violence ont tendance à être plus élevés dans le Sud et l'Ouest (Harries, 1973). Toutefois, dans les régions américaines de moindre criminalité - le Nord-Est et le Centre-Nord - les taux par habitant sont plus élevés que ceux de la Colombie-Britannique, au Canada (Harries, 1973 ; Fattah, 1973 ; Solliciteur général, 1979b), où ils atteignent le niveau national maximum.

\section{Meurtre}

Le graphique II offre pour la période qui va de 1965 à 1978 , une comparaison du taux de meurtres ${ }^{8}$ au Canada et aux Etats-Unis par 100000 habitants. On y constate que les taux américain et canadien de meurtres ont approximativement doublé pendant ce laps de temps, mais le taux américain est environ quatre fois plus élevé que celui s'appliquant à l'ensemble du Canada.

Les taux de meurtres par 100000 habitants varient néanmoins selon les Etats. En 1978 par exemple, pour le Texas, ils étaient 14 ; pour la Californie, la Floride et New York environ 11, mais il y avait sept Etats avec moins de trois par 100000 - Maine $(2,7)$, Iowa $(2,6)$, Wisconsin $(2,5)$, Minnesota $(2,0)$, Dakota du Sud $(1,9)$, New Hampshire $(1,4)$, et Dakota du Nord

7. Précisons que la majorité des délits compris dans l'indice de violence, sont des vols qualifiés. Pour détails voir \& Quelques tendances en justice pénale canadienne s, Solliciteur général du Canada, 1979a.

8. Sous le terme de meurtre on comprend (i) pour les Etats-Unis les meurtres et les homicides involontaires coupables (\& non négligents \$) et (ii) pour le Canada, les meurtres qualifiés et non qualifiés et les homicides involontaires coupables. 
GRAPHIQUE II

Taux de meurtres par 100000 habitants

Canada - Etats-Unis (1965.1978)

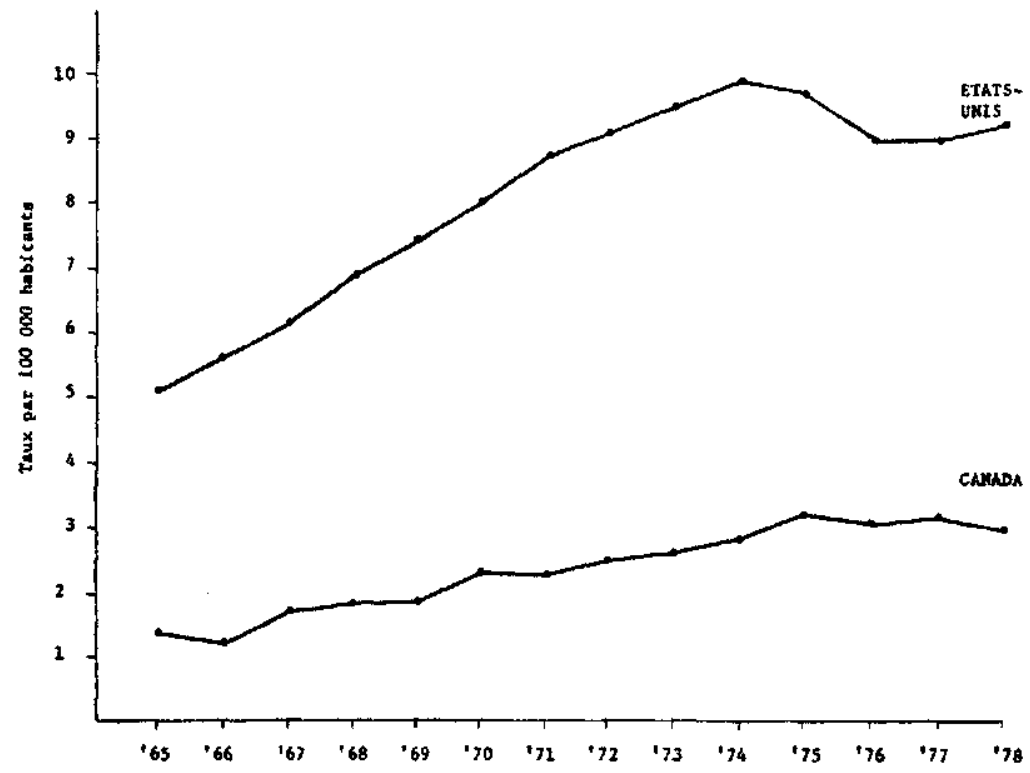

$(1,2)$. Ces derniers sont parallèles aux taux relevés pour les provinces canadiennes qui varient d'environ 1,0 pour les Maritimes à 3,0 pour la Colombie-Britannique.

On peut constater des tendances analogues dans le cas du crime de viol, où le taux des Etats-Unis est trois fois celui du Canada.

\section{Crime contre les biens}

Le graphique III montre un phénomène analogue en ce qui a trait aux délits de cambriolage et de vol qualifié. Cette fois, le taux de vols qualifiés pour les États-Unis est environ le double de celui du Canada, tandis que le taux de cambriolages n'est que de 20 à $30 \%$ plus élevé.

\section{Criminalite des affaires}

L'affaire Watergate n'a pas d'équivalent au Canada. L'inculpation d'un vice-président, d'un procureur général et d'une série 
GRAPHIQUE III

Vols qualifiés et cambriolages par 100000 habitants

Canada - Etats-Unis (1965-1978)

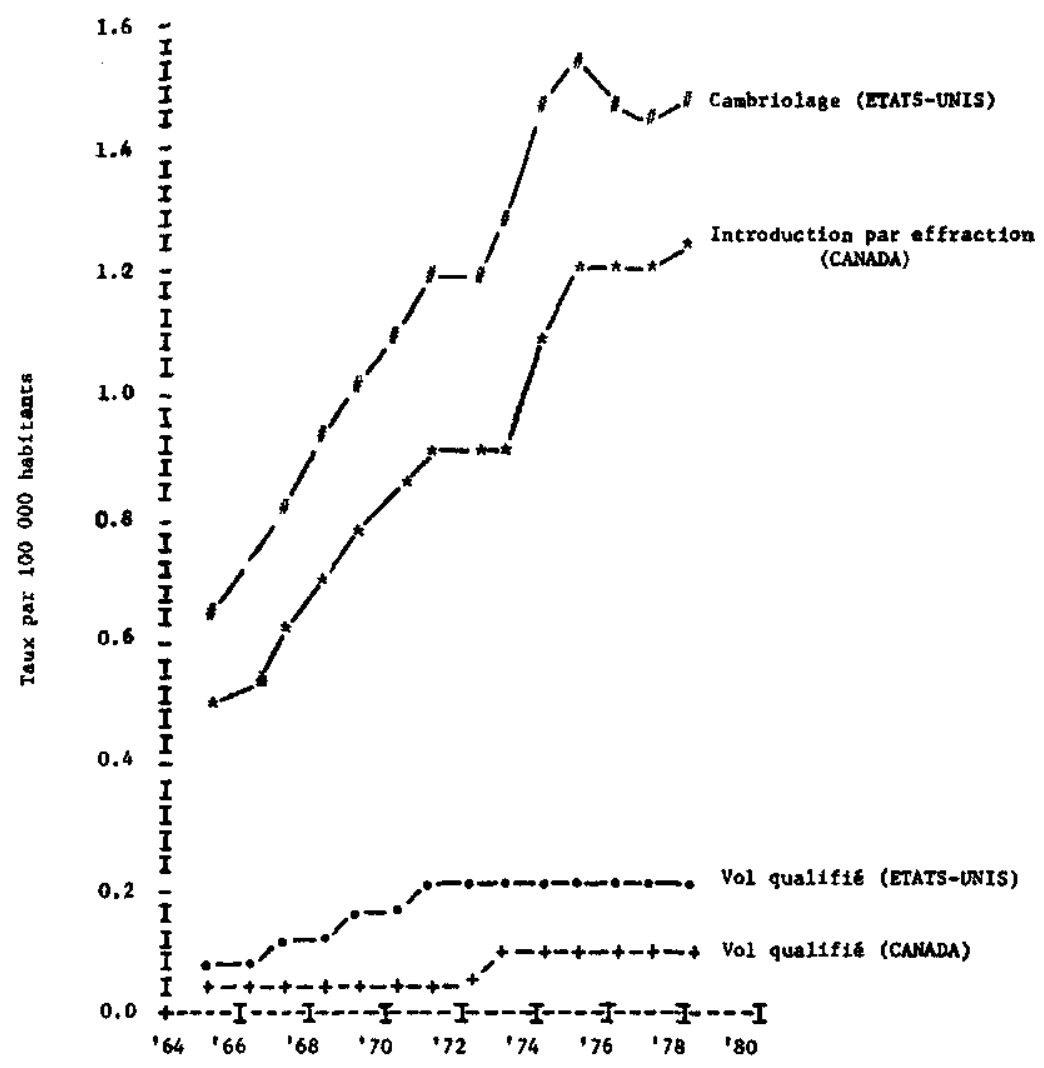

de hauts fonctionnaires a frappé tous les pays industrialisés, comme un scandale sans précédent. La brutalité et la corruption de certaines forces policières américaines et les actes de meurtre, de révolution et de torture imputés à l'occasion à la C.I.A., sont bien connus aux États-Unis.

Au Canada, le scandale > des introductions par effraction bien qu'autorisées par des supérieurs d'une grange brôlée et des fichiers copiés a été assez important - dans notre contexte politique - pour entraîner l'établissement d'une commission royale d'enquête et pour occuper les colonnes des journaux pendant plus de deux ans. 
Il est très difficile, en fait, de mesurer ce genre d'activités, comme d'ailleurs la criminalité des compagnies et celle des divers échelons gouvernementaux. Certes des opérations illicites existent et sont facilitées par des innovations technologiques ainsi que par le développement des marchés internationaux. Certes les sommes d'argent impliquées dans la criminalité des affaires sont plus élevées que celles de la criminalité traditionnelle, tandis que les coupables sont traités avec plus d'égard, tout compte fait, que les autres criminels, mais il n'en reste pas moins qu'ils présentent une menace, perçue comme emoins brutales par les citoyens parce que moins directe.

Nous ne pouvons pas mesurer la responsabilité \& criminelle \$ d'un directeur qui accepte la construction d'un réservoir d'essence dont l'explosion va tuer par la suite 100 personnes, mais diminuer à long terme le coût des voitures achetées par d'autres consommateurs de plusieurs centaines de dollars. De même, nous ne pouvons pas discuter ici, de la responsabilité d'un directeur qui laisse en usage un type d'avion dont la porte de cargaison est défectueuse en espérant protéger ainsi les emplois de plusieurs ouvriers, ou constructeurs.

Comme les Etats-Unis sont le centre de production de ces appareils et du commerce international, nous supposons donc que ce genre de criminalité y est plus répandu, mais les victimes se retrouvent autant au Canada qu'en Amérique en général, ou ailleurs.

\section{L'alcoolisme}

En 1978, on dénombrait aux Etats-Unis 591 arrestations pour « ivresse , par 100000 habitants; 478 pour conduite de voiture avec des capacités affaiblies par l'alcool; et 160 pour d'autres infractions reliées à l'alcool', soit au total $26 \%$ de toutes les arrestations. Au Canada, les données relatives aux arrestations pour le délit d' ' ivresse > ne sont pas disponibles, mais pour conduite de voiture avec capacités affaiblies par l'alcool, on dénombrait 592 infractions (le nombre d'arrestations n'est pas disponible) par 100000 habitants ${ }^{10}$.

9. F.B.I. Uniform Crime Reports, 1978.

10. Solliciteur général du Canada, 1977, p. 112-113. 
L'indice a approximativement doublé, de 1968 a 1978, pour les deux pays, mais le taux d'ivresse a diminue de $50 \%$ aux Etats-Unis. Comme la moitié environ d'accidents mortels de la route sont attribuables à une incidence d'alcool, ces données sont d'autant plus frappantes.

\section{L'usage et le commerce des stupefiants}

En ce qui a trait aux infractions relatives à l'abus de drogues, aux Etats-Unis, il y avait 304 arrestations par 100000 habitants en $1978^{11}$, comparativement aux 244 infractions par 100000 , en 1975 , pour le Canada ${ }^{12}$.

Aux Etats-Unis, $10 \%$ des détenus des prisons des États (N.C.J.I.S.S., 1974, p. 2) étaient là pour les infractions reliées aux drogues. Au Canada, 10\% des détenus des pénitenciers, condamnés à deux ans ou plus, s'y trouvaient pour des raisons semblables ${ }^{13}$.

Ces données sont difficiles à interpréter, parce qu'une infraction au Canada n'implique pas nécessairement une arrestation et les types d'emprisonnements sont différents d'un pays à l'autre, mais elles montrent qu'un pourcentage important de criminalité connue de la police, des tribunaux et de l'administration des prisons, est relié aux drogues. Quantitativement, l'incidence des drogues est moins importante que celle de l'usage d'alcool, mais aboutit à des peines de prison plus longues. D'une manière générale l'usage de certaines drogues est dix fois plus important selon les statistiques de criminalité aux États-Unis qu'au Canada et dès lors la criminalisation de ces stupéfiants est un des facteurs explicatifs des taux de la criminalité américaine par nombre d'habitants.

Les infractions relatives au code de la route

L'augmentation du parc automobile a été associé à une croissance extraordinaire du nombre d'infractions relatives aux règlements de la circulation. Selon le rapport de la Commission d'enquête présidée par le juge Ouimet ${ }^{14}, 98 \%$ d'augmentation

11. F.B.I. Uniform Crime Reports, 1978, p. 118.

12. Solliciteur général du Canada. 1977. p. 112.

13. Solliciteur général du Canada, 1977, p. 66-67.

14. Rapport du Comité canadien de la réforme pérale et correctionnelle. Imprimeur de la Reine, Canada, Ottawa, 1969. 
des condamnations au Canada, de 1901 à 1965, était due aux violations des règlements relatifs à la circulation. Ci-haut, nous avons traité des infractions au code de la route où il y avait incidence de l'alcool, mais malheureusement on ne dispose pas de données comparables pour les deux pays.

\section{Les répercussions du crime}

A partir de 1967, les Etats-Unis se sont fortement intéressés à quantifier les répercussions du crime du point de vue des pertes financières et des torts physiques. Malheureusement, le Canada n'a pas encore effectué d'études de victimisation de ce genre, bien que quelques études menées à Toronto et à Vancouver fournissent certaines données comparatives.

Les caractéristiques des effets du crime \& moyen $>$ ne different guère aux Etats-Unis de celles connues aux Canada. Comme le taux de crime par 100000 habitants est plus élevé aux ÉtatsUnis, les effets cumulatifs sont toutefois plus graves. C'est ainsi que pour 1974, par exemple, les pertes financières et les couts des blessures infligées semblent être d'un ordre analogue dans les deux pays dans les cas d'un cambriolage \& moyen * (Waller and Okihiro, 1978), pour lequel ils se situent en moyenne a $500 \$$ environ ou un total de 1,6 milliard de dollars pour les États-Unis (U.S. Department of Justice, 1979), et par conséquent à 140 millions pour le Canada. Cette évaluation augmente il va sans dire, avec le rythme de l'inflation.

Une différence qualitative entre les deux pays apparait, selon toute probabilité, en ce qui a trait aux voies de fait graves. L'usage des armes est probablement moins courant au Canada que les $22 \%$ aux Etats-Unis, mais nous ne disposons pas de données susceptibles de le préciser.

Nous n'avons pas analysé jusqu'à présent les répercussions d'ordre émotionnel qu'entraîne chez les citoyens la criminalité. Quelques études ont porté, dans les deux pays, sur les victimes de viol (Brickman, 1979 ; Burgess et al., 1974). Elles attirent l'attention sur les effets ressentis par les victimes à la suite de la menace contre leur personne : anxiété et nervosité, dépression, troubles de sommeil, pleurs, impression de solitude, difficultés de concentration, craintes intenses et rapides changements d'humeur. 
Ces traumatismes se produisent aussi dans les cas de vol qualifié et d'introduction par effraction. Une étude a été réalisée à partir d'un examen des dossiers de police relatifs aux cambriolages et aux vols qualifiés dans trois villes des Etats-Unis (Bourque et al., 1978) et une étude des cambriolages résidentiels dans la Communauté urbaine de Toronto (Waller and Okihiro, 1978).

Les symptômes ne se manifestent pas nécessairement dans chaque cas. Tandis qu'ils se produisent a la suite de la plupart des vols qualifiés et des viols, on les constate seulement dans un cas sur 20 pour l'introduction par effraction. Cette proportion de 1 sur 20 représente chaque année environ 15000 cas au Canada et près de 200000 aux Etats-Unis, ce qui dépasse le total des vols qualifiés et des viols.

Bien que les pertes financières et les traumatismes causés puissent correspondre aux Etats-Unis et au Canada en ce qui a trait aux "crimes moyens ", il n'en reste pas moins que les pertes globales et le nombre de personnes traumatisées est forcément plus élevé chez nos voisins du sud. En outre, le nombre des personnes tuées et blessées lors des délits commis à main armée est plus élevé aux Etats-Unis que chez nous.

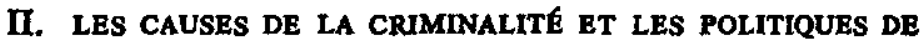 CONTRÔLE DU CRIME}

Les causes de la criminalité et ses conséquences ne peuvent être analysées uniquement à partir des graphiques basés sur les données déclarées par la police à un organisme statistique central. Nous nous proposons donc d'examiner en premier lieu la façon suivant laquelle les divers délits sont perçus par les citoyens, définis sur le plan légal et rapportés par la police.

Nous étudierons ensuite les justifications culturelles et structurelles - héritages culturels, changements démographiques, et conflits raciaux ou assimilés. Dans la troisième section, nous examinerons les explications reliées à l'environnement et la privation relative, que l'on peut difficilement séparer des questions précédentes de culture et de structure. Finalement, nous discuterons des politiques générales de contrôle du crime, qui en découlent. 


\section{A. Les recensements de la criminalité}

\section{La réaction des citoyens}

La Commission présidentielle sur l'administration de la justice aux Etats-Unis ${ }^{15}$ a souligné dans son rapport le fait qu'un grand nombre de crimes, légalement définis comme graves,

\section{TABLEAU I}

Le pourcentage de quelques types de victimisation signales d la police

(par ordre de fabllite)

\begin{tabular}{l|c|c|l}
\hline & \multicolumn{2}{|c|}{ Etats-Unis } & \multicolumn{1}{c}{ Canada } \\
\cline { 2 - 4 } & $\begin{array}{c}\text { National } \\
\text { Crime Surveys } \\
1973-1977^{*}\end{array}$ & $\begin{array}{c}\text { Ennis } \\
(1967)\end{array}$ & $\begin{array}{l}\text { Waller and Okihiro (1978) } \\
\text { Solliciteur général (1980) }\end{array}$ \\
\cline { 2 - 4 } Vol d'auto & 69 & 89 & 75 (Vancouver) \\
Cambriolage & 49 & 58 & 62 (Toronto et Vancouver) \\
Vol qualifié & 54 & 65 & 50 (Vancouver) \\
Voies de fait & 46 & 53 & 35 (Vancouver) \\
Viol & 54 & $(77)$ & - \\
\hline
\end{tabular}

* Moyenne pour la période.

ne sont pas signalés par les citoyens à la police. Le tableau I indique la façon suivant laquelle le public dénonce certaines infractions aux États-Unis et au Canada. Les estimations concernant le Canada sont limitées toutefois aux résultats de deux études systématiques portant sur des échantillons sélectionnés à Toronto (Waller and Okihiro, 1978), et à Vancouver (étude publiée par le Solliciteur général du Canada, 1980).

Pour les Etats-Unis, nous avons retenu deux périodes distinctes, soit tout d'abord l'étude primaire de Ennis (1967) qui donne les pourcentages de victimisation signalés à la police puis ceux publiés dix ans plus tard par le National Crime Survey (Sourcebook, 1979), qui contient des relevés systématiques. Bur

15. U.S. President's Commission on Law Enforcement and Administration of Justice, 1967. 
la base de ces données on constate qu'une proportion élevée de victimes ne signale pas à la police les délits concernés et cela tout aussi bien aux États-Unis qu'au Canada. C'est ainsi, par exemple, qu'aux États-Unis, il semble qu'il y a moins de crimes signalés dans les années 70 qu'auparavant, $\infty$ qui peut être attribuable, entre autres, au fait que l'assurance des biens a probablement augmenté durant cette période.

Compte tenu du fait qu'uniquement $50 \%$ des délits sont rapportés, l'importance des citoyens en tant que « déclencheurs * du système de justice pénale ressort clairement. Il ne semble pas toutefois, que les différences dans les taux de dénonciations, entre les deux pays, soient un facteur diriment en ce qui a trait aux différences des données relatives aux divers types de délits. D'autres caractéristiques propres aux États-Unis et au Canada doivent être analysées à cet égard.

\section{Les différences des définitions légales}

Comme nous l'avons déjà signalé, il est surprenant de constater que peu d'analyses ont été réalisées en vue de comparer les niveaux de criminalité des États-Unis avec ceux du Canada. L'étude canadienne portant sur le cambriolage résidentiel dans la Communauté urbaine de Toronto au Canada (Waller and Okihiro, 1978), constitue une exception à cet égard. Afin d'éviter les confusions découlant des disparités des termes légaux qui ne sont pas compatibles, dans cette étude on a adopté une définition du délit de cambriolage qui a permis des comparaisons entre Toronto, au Canada, et un certain nombre de villes des Etats-Unis où l'on avait employé une définition semblable dans des études de victimisation. On a réussi à confirmer ainsi que le taux de cambriolage résidentiel dans la Communauté urbaine de Toronto représente les deux cinquièmes environ du taux le moins élevé parmi les huit grandes villes des Etats-Unis.

Extrapoler à partir de cette étude, au niveau national, demeure difficile, néanmoins, notons, à cet égard, que le taux d'introduction par effraction dans les domiciles de Toronto, tel qu'il a été enregistré par la police, représente approximativement la moitié de celui relevé à Montréal et à Vancouver. Cela ne remet pas, cependant, en cause les statistiques sur lesquelles eśt basé le graphique III. 


\section{Les fluctuations du niveau de criminalité déclarée}

Dans un certain nombre d'études il a été démontré par ailleurs que les fluctuations considérables des niveaux de criminalité déclarée découlent de changements apportés par les administrateurs du système, tels les chefs de police. A New York, la réorganisation mené en 1950 a fait augmenter soudainement d'une année à l'autre les taux de vol qualifié de $400 \%{ }^{16}$. Une étude des détachements de la Gendarmerie royale du Canada a révélé que dans les endroits où le chef du détachement peut obtenir de l'avancement en portant son niveau de criminalité déclarée au-dessus d'un seuil donné, on constate une hausse de la criminalité dans ce secteur (Oosthoek, 1978). D'autres commentateurs ont discuté des diverses préoccupations semblables, toutefois, au cours des dix dernières années, les facteurs en question n'ont pas pu être sélectionnés de façon cohérente.

L'accroissement du nombre d'appareils téléphoniques, l'informatique et, en particulier, la centralisation des services de police ont pu contribuer à une certaine hausse du taux de la criminalité déclarée. Toutefois, quand il s'agit des villes telles que Toronto, qui n'ont pas connu de changements similaires, les taux de criminalité y ont suivi la même tendance générale d'accroissement.

En bref, la façon de recenser la criminalité ne permet pas d'expliquer cette augmentation ni les différences qu'on observe entre les deux pays. Apparemment le taux de crimes signalés a diminué légèrement et le nombre de ceux enregistrés par la police a augmenté un peu.

\section{B. La culture et la structure}

\section{Héritages culturels}

La profonde différence entre le Canada et les Etats-Unis et la plus malaisée à quantifier est celle de l'héritage culturel. C'est ainsi, par exemple, qu'Hollywood produisait des westerns symboliques des luttes des pionniers tandis qu'on tournait au Canada des films romantiques sur le rôle de la * police montée , dans le Grand Nord. Il est difficile de croire cependant que la criminalité des centres des grandes villes est influencée par l'ancien temps.

16. Skogan, 1975, p. 23 ; Wolfgang, 1968. 
La glorification du «cowboy > et des disputes résolues à l'aide des armes à feu au lieu d'une intervention de la police a des racines dans le folklore américain et la guerre civile qui a laissé des traces de suspicion face au pouvoir central et à l'État en général. Silberman (1978) prétend que la violence est aussi « américaine que Jesse James $\gg$, mais est-ce à cause des valeurs traditionnelles ou plutôt en raison d'une structure sociale discriminatoire ? Peut-on présumer à l'opposé que la déférence à l'égard de l'autorité et l'effort pour résoudre les disputes (exception faite des patinoires du hockey) par des moyens non violents sont caractéristiques de la population du Canada?

Aux Etats-Unis l'idée d'une police nationale est perçue comme le premier pas vers le totalitarisme (Caplan, 1977). Les hommes politiques américains s'inquiètent même des associations professionnelles des policiers. Ce trait national américain de donner de l'importance à la liberté de l'individu et d'éviter des concentrations du pouvoir n'a pas d'équivalent au Canada. Pour 10 de nos 12 juridictions (10 provinces et deux territoires) le principal corps de police demeure la Gendarmerie royale du Canada. Celle-ci est également un symbole national d'unité et semble constituer une sorte de protecteur de l'ordre public. Un tiers de tous les policiers canadiens font partie de la Gendarmerie royale; le deuxième tiers est divisé entre quatre autres forces policières. Les hommes politiques canadiens parlent parfois de créer une gendarmerie nationale et il est possible de prétendre que la coordination des forces policières peut réduire la criminalité au Canada. Il n'en reste pas moins que les indicateurs de - U.C.R. > qui tiennent compte des variables à caractère social ne sont pas très influencés par le déploiement des forces policières (Conklin, 1972 ; Reppetto, 1974 ; Scarr, 1973 ; Waller and Okihiro, 1978; Hirschi, 1969).

D'autre part l'importance accordée à l'individu par la culture américaine renforce le manque de liens et encourage le culte du criminel-héros. Cette glorification du criminel constitue une tendance beaucoup plus exagérée aux États-Unis qu'au Canada. D'abord le système électoral des Etats-Unis donne en soi une place importante au problème du crime. En général, les juges, les directeurs des prisons locales (dont il y a plus de 4000 avec un tiers des prisonniers en moyenne quotidienne) les procureurs sont élus tout comme les maires, les députés et les sénateurs. Chacun doit 
donc mener sa campagne avec le résultat qu'ils stimulent ainsi l'intérêt à l'égard de la criminalité, ou de l'individu criminel ce qui a des conséquences négatives. C'est ainsi, par exemple, que jusqu'à très récemment, l'attention a été détournée des soins et d'assistance devant être apportés à la victime. De plus, les médias tant la télévision que la presse écrite croient que le public est avide des descriptions des cas sensationnels et exceptionnels ainsi que des images des policiers romantiques qui traquent des criminels.

L'opinion publique à l'égard du crime semble être au Canada un peu plus conservatrice qu'aux Etats-Unis; un plus grand nombre de Canadiens que d'Américains veulent imposer la peine de mort (Fattah, 1975) et nous sommes aussi plus favorables aux longues peines d'emprisonnement que nos voisins du sud (Perspectives Canada III, p. 306).

D'une manière générale, toutefois, l'effet de l'héritage culturel sur la criminalité est difficile à évaluer. En bref, le criminelhéros et l'individualisation font plus partie de la culture américaine, et les valeurs conservatrices sont plus proches de celles du Canada, mais ces données ne semblent pas de toute façon avoir la même importance pour expliquer les variables de la criminalité que les facteurs structurels.

\section{Les changements démographiques}

C'est ainsi que les Etats-Unis comme le Canada, ont connu après la guerre une explosion des naissances qui a commencé vers 1945 et qui s'est poursuivie jusqu'en 1960 (Perspectives Canada III, 1980). Or, selon les études sur les personnes condamnées et la criminalité révélée, (Hindelang, Hirschi and Weis, 1979), les auteurs des crimes sont majoritairement des adolescents et hommes jeunes âgés de 15 à 29 ans. Il est donc à présumer qu'une hausse de la proportion de ces groupes d'âge dans la population, entraîne automatiquement une augmentation du taux de criminalité.

En 1976, l'âge moyen des Canadiens est de 27,8 ans et celui des Américains de 29 ans, donc 1,2 de différence. Au Canada l'augmentation en termes de l'importance de ce groupe a passé de $21 \%$ en 1962 à $28 \%$ en 1978 . En termes absolus le nombre des citoyens de cet âge dans les deux pays a augmenté de plus 
de $60 \%$ entre 1962 et 1978 . Les spécialistes attribuent l'accroissement du taux de criminalité de $25 \%$ aux Etats-Unis (Silberman, 1978) et $27 \%$ au Canada (Beattie, 1980) à cette croissance, ce qui signifie qu'elle est une explication partielle de la hausse de la criminalité dans les deux pays mais ne les différencie pas clairement pour autant.

Des nombreuses études criminologiques, réalisées aux ÉtatsUnis dès 1930, attribuent un rôle important à l'immigration en tant que facteur de hausse de la criminalité, la théorie étant que les Européens démunis qui arrivaient dans les grandes villes américaines avaient des difficultés à s'adapter ainsi que les Noirs qui quittaient les Etats du Sud pour aller vivre dans les villes du Nord-Est. Tant en 1961 qu'en 1971, les immigrants représentaient $5 \%$ uniquement de la population totale des Etats-Unis, tandis qu'au Canada cette proportion était de $15 \%$ (Perspectives Canada III, 1980, p. 295). L'étendue des migrations de zones rurales vers les régions urbaines est difficile à comparer entre les deux pays, mais reste fort limitée entre 1961 et 1971 en constituant même un phénomène difficile $a ̀$ isoler de l'immigration en tant que telle. Il est important de noter, cependant, que Toronto, maintenant la ville la plus peuplée du Canada, n'a pas le taux de criminalité le plus élevé par rapport à la moyenne nationale bien que sa croissance se situe parmi les plus rapides de l'Amérique du Nord et qu'un tiers de ses résidents soit né à l'extérieur du pays.

\section{Les autochtones et les problemes raciaux}

Une des caractéristiques majeures de la situation de la criminalité au Canada demeure le nombre élevé des autochtones arrêtés par la police et mis en prison. Sur le plan national, 129 adolescents par 100000 habitants sont reconnus coupables; pour les autochtones, ce taux passe à 353 pour 100000 autochtones. Au niveau adulte, 235 par 100000 autochtones sont incarcérés, mais au total ils représentent $9 \%$ de la population carcérale adulte et seulement 3 à $3,5 \%$ de la population nationale ${ }^{27}$. Les infractions qu'ils commettent sont de nature très variée, allant de la violation des règlements de permis de pêche au meurtre, mais se concentrent de façon disproportionnée dans les catégories

17. Affaires Indiennes et du Nord (1980). « Les Indiens : situation actuelle s, 
telles que les cambriolages, l'aicoolisme et la violence. Cette tendance est aggravée sans conteste par des taux élevés de chômage, par la pauvreté et des conflits culturels avec l'ensemble de la société canadienne ${ }^{18}$. La surreprésentation des autochtones dans les prisons peut être due également à l'absence d'un choix de programmes de traitements de semi-liberté dans les communautés isolées où ils vivent.

Le même phénomène se produit aux Etats-Unis et c'est ainsi, par exemple, qu'au Dakota Sud, le taux de 100000 Amérindiens incarcérés est de 545 en rapport avec 83 pour les Blancs (chiffre approximatif recalculé de Dunbaugh, 1979), mais les proportions sont moins frappantes, il va sans dire, quand il s'agit de la population totale des Américains comparativement au groupe total des Amérindiens, limité par définition.

Aux Etats-Unis, la criminalité est fortement reliée aux phénomènes raciaux. Certes la proportion des hispaniques en prison n'est que légèrement plus élevée que celle de la population en général, mais les Noirs par contre sont surreprésentés. En effet, bien qu'ils ne constituent que $12 \%$ de la population des Etats-Unis, $47 \%$ des personnes dans les prisons d'États ou fédérales (N.C.J.I.S.S., 1979a) ; 41\% dans les prisons locales (N.C.J.I.S.S., 1980c) ; 34\% de mineurs en détention sont des Noirs (N.C.J.I.S.S., 1979b). Les indicateurs par rapport à la population totale sont encore plus dramatiques, quand on les compare avec ceux de l'Afrique du Sud, puisque aux États-Unis on les évalue à 100 pour 100000 quand il s'agit des Blancs et à 600 pour 100000 pour les Noirs, tandis qu'en Afrique du Sud les proportions respectives sont de 86 et de 790 .

Bien que le pourcentage des Amérindiens soit trop faible pour influencer les indicateurs totaux, les phénomènes raciaux sont un facteur important dans la différence qui existe entre les taux de criminalité du Canada et des États-Unis.

\section{L'usage des stupéfiants}

Il y a plusieurs évaluations du nombre de personnes qui s'adonnent à l'hérö̈ne en violation des lois en vigueur. Aux États-Unis elles varient, selon les méthodes d'analyse utilisées, de

18. Affaires Indiennes et du Nord (1980), op. cir. 
115 à 275 par 100000 habitants (Silberman, 1978, p. 1975 ; Gandossy et al., 1978, p. 93-108), tandis qu'au Canada, Armstrong et Turner $(1976$, p. 517$)$ suggèrent 14 pour 100000 , soit un taux représentant moins du dixième de celui de nos voisins du sud.

Plusieurs auteurs (Repetto, 1974 ; Gandossy et al., 1978) prétendent que les narcomanes doivent voler pour acheter la drogue et que, par conséquent, quand le prix augmente le niveau de criminalité s'accroît. Sans parler du commerce illicite en tant que tel, l'importance de consommation d'héroïne aux Etats-Unis permet de présumer qu'un tiers des vols des biens (Gandossy $\boldsymbol{e}$ al., 1978, p. 106) peut être relié à son usage comme à celui des autres stupéfiants.

Selon Gandossy et al. (1978), une augmentation de $10 \%$ du prix de l'héroïne est associée à l'accroissement de $1 \%$ de la criminalité.

Toutefois ce qui importe surtout ce ne sont pas les ordres de grandeurs, mais le fait que la consommation est beaucoup plus élevée aux États-Unis qu'au Canada et qu'au cours des 15 dernières années, elle s'est diversifiée tout en ne cessant pas d'augmenter.

\section{Les armes d feu}

La tradition des armes à feu remonte aux Etats-Unis à la guerre civile et est encouragée par la conviction sanctionnée dans la Constitution américaine du droit au port d'arme pour assurer sa propre défense. La différence entre le Canada et les États-Unis est très prononcée à cet égard. Bien qu'il y ait 70000 armes à feu par 100000 citoyens aux Etats-Unis et 50000 au Canada, on compte seulement 4000 armes à feu courtes par 100000 Canadiens, par rapport à 20000 pour les États-Unis (Solliciteur général du Canada, 1976 ; Stenning et Moyer, 1979). A première vue, cela devrait constituer un important facteur contribuant à la différence qui existe entre les deux pays en ce qui a trait aux taux de meurtres, de vols qualifiés et de voies de fait. En effet selon les travaux de Pierce et Bowers (1979), entre autres, la réduction de ce genre de criminalité est liée a un meilleur contrôle des armes à feu. 
Donc, la tradition relative aux armes à feu, ainsi que l'importance des toxicomanes et les structures raciales différencient les Etats-Unis du Canada d'une façon très marquée et sont tous probablement des facteurs du plus haut niveau de la criminalité américaine par rapport à celle de notre pays. Les changements démographiques, qui ont contribué pour $25 \%$ environ à l'augmentation des taux de criminalité, n'ont fait en outre qu'accentuer les disparités.

\section{L'environnement et les conditions socio-économiques \\ L'environnement urbain}

En ce qui a trait à l'environnement urbain un élément dramatique, pratiquement inconnu au Canada, intervient aux Etats-Unis. C'est ainsi que la criminalité américaine s'est concentrée dans les ghettos, les secteurs urbains délabrés, tels le Bronx et Harlem, où des blocs entiers de logements ont été abandonnés par leurs habitants désireux de fuir le centre de la grande ville en proie aux incendies, aux crimes résidentiels et à la détérioration des services publics. Les conséquences de cette situation de l'environnement urbain sont reconnues dans les programmes de prévention de la criminalité (U.S. Department of Housing and Urban Development, 1979). Malgré les difficultés de définition des ghettos, certains auteurs tel Silberman $(1978$, p. 36) laissent entendre que les taux actuels de chômage comme de naissances dans les centres-villes des grandes cités américaines diffèrent beaucoup de la tendance générale. En 1976, parmi les Noirs pauvres, $48 \%$ étaient âgés de moins de 14 ans comparativement à $24 \%$ représentés par ce groupe dans l'ensemble de la population.

D'autre part, l'analyse de la notion d'espace défendable (Newman, 1972 ; Waller and Okihiro, 1978 ; Mayhew, 1979) a provoqué un vif intérêt à l'égard des études concernant l'incidence de la conception des logements urbains sur la perpétration de crimes. Cohen et Felson (1979) ont parlé du style de vie ; Hindelang (1978) dans son analyse sur les études de victimisation aux États-Unis, s'est penché sur un phénomène analogue.

Il est possible qu'un autre facteur contribuant à la difference des taux de criminalité entre le Canada et les États-Unis se rattache à l'aménagement du milieu. Une fois de plus, nous nous reportons à la comparaison détaillée de Toronto, Washington et 
Boston, effectué dans nos propres études comme dans certaines autres (Waller and Okihiro, 1978; Reppetto, 1974 et Scarr, 1973), qui semble démontrer que d'une façon globale la conception des appartements et des maisons, a une incidence sur les cambriolages résidentiels. Il est possible qu'il y ait beaucoup plus d'Américains que de Canadiens qui vivent dans des maisons à appartements de plusieurs étages où les liens communautaires qui réduisent les occasions du crime, sont particulièrement faibles ou inexistants.

\section{Disponibilité des biens}

Les Américains ont plus de téléphones, de télévisions, et de voitures : $61 \%$ des ménages aux Etats-Unis comparativement à 43\% au Canada ont un, ou plus d'un téléviseur en couleur (Perspectives Canada III, p. 109). Au Canada le pourcentage a augmenté de $10 \%$ en 1965 (p. 274) et le nombre de ménages avec deux postes de radio est passé de $41 \%$ (1965) à $73 \%$ (1978). En ce qui a trait aux voitures $32 \%$ des ménages américains et $20 \%$ des couples canadiens ont deux automobiles ou plus. Comme on dénombre aux États-Unis 59800 voitures par 100000 habitants, en comparaison de 37400 pour le Canada, le taux de vol d'autos est plus faible par voiture aux États-Unis.

Selon Cohen et Felson (1979) l'augmentation de la criminalité aux Etats-Unis s'explique, entre autres, par la croissance de la quantité des biens disponibles, leur transportabilité et leur répartition. Ils citent des auteurs, tel Gould (1969) qui a démontré que l'augmentation du nombre des billets de banque et des voitures, entre 1921 et 1965, semble être reliée à l'accroissement du nombre de vols de banques entre autres. Ils constatent aussi que :

a) pour chaque million d'heures où les voitures étaient stationnées, il y avait deux voitures volées dans les résidences, mais 55 dans les stationnements et les rues;

b) pour chaque $100 \$$ dépensés, on a volé $12 \not$ des meubles et des biens durables non électroniques mais $6,84 \$$ d'appareils électroniques ;

c) pour 100 ménages, le taux de victimisation était de 168 pour une personne seule et 326 pour des familles de six personnes ou plus. 
Ils ont démontré aussi que l'augmentation du pourcentage des femmes au travail qui a été de $31 \%$ de 1960 à 1970 a été associée à l'accroissement du nombre de résidences où il n'y avait personne le matin. Ce pourcentage a passé au Canada de $21 \%$ en 1965 à $26 \%$ en 1976 , pour les femmes de 25 ans et plus (Perspectives Canada III, p. 89), soit un taux de $23 \%$. Selon nos propres études (Waller and Okihiro, 1978), l'absence des gens au foyer est associée à une plus grande probabilité de cambriolage, ce qui confirme la thèse de Cohen et Felson selon lesquels il en découle l'augmentation des cambriolages des résidences.

\section{Les liens sociaux}

Des études sur les jeunes en Californie de Hirschi (1969) et à Montréal de Leblanc et Fréchette (1979), concernant la population scolaire, comme nos propres travaux (Waller, 1974) sur les prisonniers libérés des pénitenciers fédéraux au Canada, indiquent que les liens d'engagements communautaires sont un facteur important dans la prévention de la criminalité. Cest ainsi qu'une personne qui a un travail, une famille, des amis en général non criminels et quelques biens est engagée dans la société et a d'autant plus de chance de ne pas récidiver (Matza, 1969) ou de devenir délinquante. À l'opposé, un adolescent qui quitte sa famille et l'école sans avoir un travail ou une famille à lui, perd les liens susceptibles de l'éloigner d'une carrière criminelle.

On ne dispose que d'indicateurs généraux, susceptibles de montrer la répartition de ce phénomène au Canada et aux ÉtatsUnis.

Toutefois, les taux bruts de chômage, au Canada et aux Etats-Unis semblent être du même ordre. Le taux de chômage total au Canada a monté au cours des années 70 . En 1978, il a été plus élevé que le taux américain, dépassant $8,2 \%$ pour les personnes de 15 ans et plus, en comparaison avec $6 \%$ pour celles âgées de 16 ans et plus aux Etats-Unis (Perspectives Canada III, p. 297). Le taux de chômage est encore plus important pour le groupe de 16 à 24 ans, n'ayant terminé que 4 ans d'école secondaire. Ce taux aux Etats-Unis est de $12 \%$ pour les Blancs, mais de $24 \%$ pour les Noirs (U.S. Social Indicator, 1977, p. xxvii). 
Au Canada il est de 15\% (Perspectives Canada III, 1980, p. 91), mais de $24 \%$ pour les jeunes qui n'ont pas dépassé le niveau des études primaires.

En 1977, le taux de mariages aux États-Unis est de 1010 par 100000 habitants soit un peu plus élevé que celui du Canada qui est de 820 par 100000 (Perspectives Canada III, 1980).

Toutefois aux États-Unis comme au Canada le taux des mariages a diminué de 1971 à 1977 , ce qui permet de présumer que moins de couples sont désormais unis dans les deux pays par ce genre de liens. Le taux brut de divorces a augmenté et aux États-Unis constitue presque le double de celui du Canada (Perspectives Canada III, 1980) ; mais cette donnée ne s'applique pas en particulier, il va sans dire, aux jeunes de 15 à 25 ans.

La proportion des ménages «libres 》 a augmenté de 1960 à 1970 de 31\% aux États-Unis (Cohen et Felson, 1979), et au Canada de 40\% environ de 1966 (15\%) à 1976 (21\%) (Perspectives Canada III, p. 22).

Une autre mesure de lien entre la criminalité et les statistiques générales de la population se situe au niveau du nombre de personnes inscrites dans les institutions d'enseignement postsecondaire. Aux Etats-Unis, ce groupe représentait approximativement $27 \%$ de la population, de 18 à 24 ans et au Canada $21 \%$ environ.

Les forces armées représentent une autre forme d'engagement ; les Etats-Unis sont intervenus dans d'importants conflits durant cette dernière période. En 1966, on y comptait près de 1600 membres des forces armées par 100000 habitants, en comparaison à un peu plus de 500 au Canada. En 1975, on constate toutefois une baisse soit 1000 par 100000 habitants aux Etats-Unis et 350 par 100000 habitants au Canada.

D'une manière générale, on peut prétendre sur la base de certaines de ces statistiques que quand les liens interpersonnels, familiaux et de travail se relâchent cela peut avoir dans les deux pays une incidence sur l'augmentation de la criminalité. Toutefois autant cela semble être partiellement démontré aux États-Unis, singulièrement pour les populations noires et les mineurs, ou des sujets très jeunes, autant tel n'est pas le cas pour le Canada. 


\section{Disparités de niveau de vie}

Lo niveau de vie de beaucoup de gens a continué à s'améliorer dans les deux pays, mais d'une façon différentielle. Le revenu médian d'une famille a augmenté de $55 \%$ au Canada, mais seulement de 19\% aux Etats-Unis (Perspectives Canada III, 1980, p. 304). En 1977, ce revenu annuel était de $14969 \$$ au Canada et de $12666 \$$ aux Etats-Unis. Cependant le taux de chômage des groupes âgés de 16 à 24 ans et ayant quatre années d'école secondaire était aux États-Unis de $12 \%$ pour les Blancs, mais de $24 \%$ pour les Noirs.

Selon Merton (1977), comme selon d'autres, une prospérité accrue ne réduit pas la criminalité parce que celle-ci n'est pas associée à la pauvreté en tant que telle, mais à la pauvreté relative - ressentie ». Au fur et à mesure que s'élevait le niveau de vie au Canada et aux États-Unis et que se développait la consommation d'énergie et la possession de certains biens, tels les voitures, les téléphones ou encore les télévisions, on a vu croître les attentes générales des citoyens en ce qui a trait à l'accès au confort de ce type. Les déclarations des hommes politiques, en particulier, ont encouragé tout le monde à croire qu'un emploi et un bon niveau de vie sont accessibles à tous. Or pour les jeunes qui passent de l'école au marché du travail cela peut être difficile à réaliser dans l'immédiat.

Les contextes socio-économiques des Etats-Unis et du Canada diffèrent cependant sur le plan des prestations et de divers services sociaux, entre autres, assurés par l'État en vue de réduire les disparités. En 1975, par exemple, 75\% des dépenses de la santé au Canada, en comparaison de $43 \%$ aux États-Unis, ont été payées par le gouvernement (Perspectives Canada III). Il n'est pas de notre propos ici de comparer en détails les prestations des services gouvernementaux du bien-être social, il nous suffit donc de remarquer qu'au Canada environ 32\% du produit national brut est versé à l'État sous forme d'impôts par rapport à $30 \%$ aux Etats-Unis. La défense par ailleurs, un important poste budgétaire, n'atteint qu'environ un tiers du niveau des dépenses de l'État canadien par habitant ce qui permet l'utilisation des fonds disponibles pour d'autres fins. Apparemment, une large part de ces fonds publics servent au paiement des allocations familiales, des prestations d'assurance-chômage et d'assurance- 
maladie, destinées à égaliser les chances ou tout au moins à réduire des écarts. Les modes de perception de l'impôt personnel au Canada par rapport aux États-Unis (Finances, 1978), demeurent un autre élément important.

\section{Les politiques de contrôle de la criminalité}

\section{Les ressources de la justice pénale}

L'accroissement des dépenses de la justice pénale, tant au Canada qu'aux États-Unis, a été considérable durant les années 60 et 70 . Elles ont atteint un total de 2 milliards 600 mille dollars en 1977-1978 au Canada (Demers, 1980) et 24 milliards en 1978 aux Etats-Unis (N.C.J.I.S.S., 1980). Compte tenu de la population, soit 23 millions, 500 mille habitants contre 218 millions, 500 mille habitants (Perspectives Canada III, 1980), le Canada a un taux de dépenses par habitant de $110 \$$ équivalent à celui de 109\$ aux États-Unis (sans tenir compte des taux de change). Ceci représente $327 \$$ par ménage moyen.

Une partie élevée des dépenses de la justice pénale est consacrée, dans les deux pays, aux services policiers. Au Canada, en 1977 , les estimations indiquaient une proportion d'environ deux tiers (\& Quelques tendances de la justice pénale canadiennes, 1977), tandis qu'aux États-Unis le chiffre correspondant était de $56^{19}$. Ainsi donc, le Canada, avec un taux de criminalité apparemment plus bas, dépense $10 \%$ de plus au titre des services policiers.

Si nous comparons le nombre d'agents de police par 100000 habitants, nous constatons que les Etats-Unis, en 1962, avaient un taux de 180 en comparaison de 170 au Canada. En 1976, ces taux avaient atteint 290 et 280 respectivement (\& Quelques tendances de la justice pénale canadienne s, 1979). Il y a donc eu augmentation du nombre d'agents de police par personne, durant la période où la criminalité a monté, mais cette croissance demeure quasi similaire aux Etats-Unis et au Canada. Les dépenses effectuées pour les services policiers, qui semblent par

19. Le montant des dépenses affectées à la police est de $54 \%$ (N.C.J.I.S.S., 1980), mais selon les pratiques américaines certains procureurs emploient leurs propres enquêteurs. Les dépenses totales effectuées à cet égard par les procureurs sont de $5 \%$ (Sourcebook, 1978, p. 45) et de façon arbitraire, la moitié de ces dépenses a été incluse dans les dopnées relevées pour les Etats-Unis. 
contre plus élevées au Canada qu'aux États-Unis, n'ont pas d'incidence pour autant sur l'important écart qui existe entre les taux de criminalité des deux pays. L'hypothèse selon laquelle l'augmentation du nombre de policiers a un effet proportionnel de dissuasion sur le crime ne semble pas se confirmer, non plus, puisque le niveau de criminalité est plus bas au Canada. A partir de là, nous pouvons aussi rejeter les raisonnements selon lesquels plus il y a d'agents de police, plus le nombre de délits augmente puisque les agents en constatent une quantité plus élevée.

Le Canada a dépensé, en 1977, 551 millions de dollars pour des services correctionnels, en comparaison avec 5 milliards de dollars aux Etats-Unis (« Quelques tendances de la justice pénale canadienne », 1979 ; N.C.J.I.S.S., 1980). Le Canada et les ÉtatsUnis ont donc dépensé un montant semblable par citoyen (sans tenir compte des taux de change). Nos voisins du sud comptent toutefois, près de 200 détenus par 100000 habitants en comparaison avec 100 pour le Canada et ils ont aussi 509 délinquants adultes sous surveillance dans la collectivité, en comparaison avec 248 par 100000 habitants au Canada (s Quelques tendances de la justice pénale canadienne $», 1979)$. Une fois de plus nous pouvons remettre en cause l'hypothèse, que le niveau de criminalité va être plus bas, si le nombre de personnes contrôlées par le système correctionnel est plus élevé.

Il semble, en somme, que l'analyse des dépenses et des effectifs du système de la justice pénale ne fournit pas les explications suffisantes quant aux variations des niveaux de criminalité dans les deux pays. On sait également ( Quelques tendances de la justice pénale canadienne $\gg, 1979$, p. 31-33) grâce aux études sur les effets d'augmentation des effectifs de la justice pénale que si elle ne s'accompagne pas d'une meilleure organisation, elle n'a pas d'effets sur les taux de criminalité dans les zones et régions concernées.

Les solutions autres que celles prévues par la justice pénale

Tant aux Etats-Unis qu'au Canada (Shearing et Farnell, 1977, * Quelques tendances de la justice pénale canadienne s, 1977), les employés des organismes de sécurité privés sont maintenant plus nombreux que les agents officiels de l'application de la loi. Cette augmentation, qui s'est produite au cours des dix 
dernières années a été encore plus prononcée que celle des taux de croissance des services policiers.

Les années 70 ont également vu se développer les programmes communautaires de prévention du crime, mis en œuvre principalement sur l'initiative des services de police. Bien qu'il n'existe pas de données précises, le nombre de personnes impliquées aux États-Unis dans les programmes de surveillance des quartiers dépasse probablement de beaucoup celui du Canada. Le développement des services américains d'aide aux victimes a également précédé celui de notre pays. Ces initiatives, toutefois, tout en étant très nécessaires, n'ont pas eu d'incidence sur la quantité de crimes commis.

\section{Les politiques criminelles}

Parallèlement, en 1968, le gouvernement fédéral des ÉtatsUnis a cré le Law Enforcement Assistance Administration dont les efforts étaient orientés en vue de réduire la criminalité et ses conséquences, rendre plus équitable et plus humaine l'appiication de la justice pénale, et instaurer des meilleures conditions de travail à l'intérieur du système. À son point culminant, en 1976, ce programme a mobilisé presque un milliard de dollars, ou près de $5 \%$ des dépenses affectées à la justice pénale. En général, on distribuait des fonds aux États qui s'en servaient le plus souvent pour compléter leurs anciens programmes, plutôt que pour opérer des changements structurels profonds.

En décembre 1979, on a adopté une nouvelle loi qui avait pour objectif l'affectation des fonds à la mise sur pied des programmes plus efficaces ce qui devait permettre de renforcer les quelques réussites des dix années précédentes. Seattle, par exemple, avait utilisé les fonds pour élaborer des programmes rationnels, axés sur des problèmes précis (Waller, 1979), qui ont réduit les cambriolages résidentiels d'environ $50 \%$ dans les secteurs où le taux était élevé (Matthews, 1976), et qui ont permis de diminuer, selon les évaluations scientifiques objectives, les vols qualifiés dans les magasins.

Malheureusement, ni le Canada ni les Etats-Unis n'ont encore adopté l'approche fondamentale choisie à Seattle - un système que les conseils d'administration, les vérificateurs généraux, les hommes d'affaires soucieux d'efficacité, les hommes de 
science et beaucoup d'autres ont recommandé comme moyen de résoudre certains problèmes de criminalité. Si la criminalité est plus élevée en 1980 qu'en 1970 aux Etats-Unis comme au Canada, c'est parce que les facteurs ont changé et que les politiques gouvernementales n'ont pas été axées sur une intervention pourtant possible. Les décès, causés par la tuberculose ou la polio, ont varié selon des facteurs historiquement déterminés dans différents pays, mais leur nombre a été restreint avec le temps, grâce à la volonté, à la détermination et à un processus logique de solution des problèmes adoptés par les pouvoirs publics.

Déçus par dix années de mauvaise utilisation de fonds et les nécessités d'un équilibre budgétaire, les États-Unis battent maintenant en retraite en ce qui a trait à leurs programmes de lutte contre la criminalité, alors qu'ils étaient probablement très près d'une stratégie leur permettant de réaliser des progrès. Le Canada a peut-être quelque chose à apprendre de cette expérience qui a coûté quelque dix milliards de dollars, et cela peut permettre d'élargir l'écart entre les deux pays, en ce qui concerne leurs taux de criminalité.

\section{CONCLUSION}

L'image de la criminalité qui se dégage de cette comparaison entre les Etats-Unis et le Canada est une image de croissance, mais d'une croissance qui débute et se termine avec des intensités différentes. Les écarts d'il y a dix années sont demeurés proportionnels à ceux d'aujourd'hui. Des délits graves, comme le meurtre, le viol et le vol qualifié, sont beaucoup plus fréquents par habitant, aux Etats-Unis qu'au Canada. Le taux des infractions contre les biens est également plus élevé. Donc les citoyens américains sont plus souvent victimes de pertes de propriété, de sévices corporels dus aux assauts à main armée en particulier et de traumatismes émotionnels provoqués par la criminalité, que les Canadiens. Les efforts de compensation pour ces sévices restent cependant inadéquats dans les deux pays.

En ce qui a trait aux infractions dites * sans victime \$, les violations des lois relatives à l'usage d'alcool et a la conduite des véhicules automobiles sont considérées comme très graves, bien que certaines juridictions en Amérique du Nord aient découragé la police d'arrêter et de poursuivre les ivrognes ordinaires. Vers la fin des années 60 à 70, on a assisté à une augmentation des con- 
damnations relatives à l'usage de drogues, mais elle est toujours moins importante que pour l'abus d'alcool. Nous n'avons pas abordé systématiquement ici la criminalité économique, il nous est donc impossible de dégager des conclusions à ce propos.

Les explications de ces tendances sont multiples et pour expliquer la croissance de la criminalité qui est commune aux deux pays, il convient de tenir compte des changements démographiques comme l'augmentation des biens disponibles ou encore des frustrations qui en découlent pour certains individus ou groupes. Ce qui est moins facile à préciser mais néanmoins fort important c'est la mesure de l'incidence du manque de liens sociaux surtout quand il s'agit des jeunes en transition entre l'école et le marché du travail.

Les justifications les plus importantes du fait que les taux de criminalité sont plus élevés aux États-Unis résident dans leur héritage culturel de violence, de l'usage des stupéfiants, l'existence des ghettos raciaux, pauvres et abandonnés ainsi qu'à la tolérance face à la possession des armes à feu courtes. Au Canada, les problèmes des autochtones se détachent en tant que facteur important des taux de criminalité, mais proportionnellement leur poids est moindre.

La criminalité aux Etats-Unis résulte en grande partie des problèmes structurels vécus par des Noirs qui ne réussissent que difficilement à l'école, vivent des problèmes familiaux et manquent d'emplois, abusent des drogues et glissent ainsi fatalement vers la délinquance. Le nombre des armes à feu en circulation, la glorification des actes criminels, la mobilité des biens et des personnes, ainsi que l'anonymat qui peut protéger ces déplacements, sont autant d'éléments aggravants.

Avec l'accroissement de la prospérité et l'instauration de l'État-providence, les dépenses de la justice pénale ont augmenté dans les deux pays. Malgré la différence des taux de criminalité, nous avons autant de policiers et de gardiens de prisons par rapport à notre population que les Américains, mais aux ÉtatsUnis on a recours beaucoup plus souvent à l'emprisonnement qu'au Canada, tout en dépensant $50 \%$ de moins par détenu. Il est difficile, comme on le voit d'établir des relations entre ces différences et les variables de la criminalité. 
Vu le rythme de croissance de la délinquance et des dépenses qui s'y rattachent, le fait le plus frappant demeure l'absence de politiques poussées et efficaces pour enrayer l'essor de la criminalité. Le problème demande une action sociale en vue de promouvoir une société ouverte à tous, pouvant diminuer les sentiments de frustration, augmenter les liens interpersonnels et interprofessionnels et perfectionner les méthodes de protection des groupes-cibles tout en réduisant le culte positif ou négatif du criminel.

En dernière analyse, les contrastes relevés a travers le temps, les taux de la criminalité canadienne et américaine devraient selon nous stimuler les études ayant pour but l'élaboration des programmes pleinement efficaces susceptibles d'entraîner une diminution réelle de la délinquance et par conséquent, de la victimisation qui en découle.

TABLEAU III *

Index comparatif des infractions avec violence déclarées par la police par 100000 habitants Canada - Etats-Unis (1964-1978)

\begin{tabular}{ccc}
\hline Annce & Canada & Etats-Unis \\
\hline 1964 & 40 & 191 \\
1966 & 39 & 220 \\
1968 & 54 & 298 \\
1970 & 71 & 364 \\
1972 & 72 & 401 \\
1974 & 98 & 461 \\
1976 & 110 & 460 \\
1978 & 107 & 487 \\
\hline
\end{tabular}

- Données statistiques relatives aux graphiques I à III. 
TABLEAU IV

Taux de meurtres par 100000 habitants

Canada - Etats-Unis (1965-1978)

\begin{tabular}{ccc}
\hline Anné & Canada & Etats-Unis \\
\hline 1965 & 1,2 & 5,1 \\
1966 & 1,1 & 5,6 \\
1967 & 1,4 & 6,1 \\
1968 & 1,5 & 6,8 \\
1969 & 1,8 & 7,3 \\
1970 & 2,2 & 7,9 \\
1971 & 2,2 & 8,6 \\
1972 & 2,4 & 9,0 \\
1973 & 2,5 & 9,4 \\
1974 & 2,7 & 9,8 \\
1975 & 3,1 & 9,6 \\
1976 & 2,9 & 8,8 \\
1977 & 3,0 & 8,8 \\
1978 & 2,8 & 9,0 \\
\hline
\end{tabular}

TABLEAU V

Vols qualifies et cambriolages par 100000 habitants Canada - Etats-Unis (1965-1978)

\begin{tabular}{lccccc}
\hline & \multicolumn{2}{c}{ Canada } & \multicolumn{2}{c}{ Etats-Unis } \\
\hline Année & Vol qualifié & $\begin{array}{c}\text { Introduction } \\
\text { par effraction }\end{array}$ & Vol qualifie & Cambriolage \\
\hline 1965 & 28 & 491 & 71 & 653 \\
1966 & 29 & 510 & 80 & 711 \\
1967 & 35 & 586 & 102 & 814 \\
1968 & 41 & 690 & 132 & 932 \\
1969 & 48 & 770 & 148 & 984 \\
1970 & 55 & 834 & 172 & 1085 \\
1971 & 52 & 874 & 188 & 1164 \\
1972 & 54 & 876 & 181 & 1141 \\
1973 & 60 & 898 & 183 & 1223 \\
1974 & 76 & 1044 & 209 & 1438 \\
1975 & 94 & 1148 & 218 & 1526 \\
1976 & 87 & 1167 & 196 & 1439 \\
1977 & 84 & 1164 & 187 & 1411 \\
1978 & 84 & 1186 & 191 & 1424 \\
\hline
\end{tabular}




\section{BIBLIOGRAPHIE}

AFFAIRES INDIENNES ET DU NORD (1980) : a Les Indiens : situation actuelle $"$, Ottawa.

ARMSTRONG, J.D. and R.E. TURNER (1976) : - Special Problem Offenders : Alcoholics, Drug Abusers, Sex Offenders s, Chapter 18, in : W.T. McGrath (ed.), Crime and its Treatment in Canada, Toronto, MacMillan, 2nd Edition.

BIDERMAN, A.D. (1966) : - Social Indicators and Goals s, in : R.A. Bauer (ed.), Social Indicators Research, Dordrecht, Holland, D. Reidel Pub. Co.

BEATTIE, R. (1980) : * The Effect of the Changing Population on Crime in Canada *, Ottawa, Solicitor General of Canada, Statistics Division, unpublished preliminary report.

BOURQUE, B.B., G.B. BRUMBACK, R.E. KING and L.O. RICHARDSON (1978) : Crisis Intervention : Investigating the Need for New Applications, Washington (D.C.), American Institutes for Research.

BRICKMAN, J., J. BRIERE, M. WARD, M. KALEF and A. LUNGEN (1979) : " Preliminary Report of the Winnipeg Rape Incidence Project ", Paper presented at the Canadian Psychological Association, Quebec, June 1979.

BURGESS, A.W. and L.L. HOLMSTROM (1974) : Rape : Victims of Crisis, Bowie, Maryland, Robert J. Brady Company.

CAPLAN, G. (1973) : Reflections on the Nationalization of Crime, 1964-1968 ,, Social Order, Arizona State University Law Journal, vol. 1973, no 3 .

CLOWARD, R.A. and L.E. CHLIN (1960) : Delinquency and Opportunity : A Theory of Delinguent Gangs, New York, Free Press.

COHEN, L.E. and M. FELSON (1979) : Social Change and Crime Rate Trends ", American Sociological Review, vol. 44, p. 528-608.

CONKLIN, J.E. (1972) : Robbery and the Criminal Justice System, Toronto, Lippincott.

COURTIS, M. (1970) : * Attitudes to Crime and the Police in Toronto *, Toronto, Centre of Criminology, Research Report.

DEMERS, D.J. (1979) : Criminal Justice Administration Expenditures in Canada : An Examination of Recent Trends, Ottawa, Ministry of the Solicitor General, forthcoming.

DUNBAUGH, F.M. (1979) : * Racially Disproportionate Rates of Incarceration in the United States 3, Prison Law Monilor, Washington, vol. 1, no 9, March, 1979.

ENNIS, P. (1967) : Criminal Victimization in the United States : A Report of a National Survey, Chicago, Ill., National Opinion Research Center, University of Chicago, Field Survey, 2.

EVANS, J.L. et G.J. LEGER (1978) : * Les enquêtes sur la victimisation au Canada s, Ottawa, Solliciteur général, Division de la recherche, Document de travail.

FATTAH, E.A. (1973) : E Étude de criminologie Ecologique ", Montréal, Université de Montréal, École de criminologie. 
FATTAH, E.A. (1975) : . Les Canadiens et la peine de mort : étude d'une attitude sociale ", Rapport non publić, Ottawa, Solliciteur général du Canada, Division de la recherche.

FARNELL, M.B. and C.D. SHEARING (1977) : Private Security : An Examination of Canadian Statistics, 1961-1971, Toronto, Center of Criminology, University of Toronto.

FRIEDENBERG, E.Z. Deference to Authority : The Case of Canada, New York, Sharpe.

GANDOSSY, R.P., J.R. WILLIAMS, J. COHEN and H.J. HARWOOD (1980) : Drugs and Crime. A Survey and Analysis of the Literature, Washington, U.S., Department of Justice, National Institute of Justice.

GOTTFREDSON, M., N. PARISI, M. HINDELANG and T. FLANAGAN (1978) : Sourcebook of Criminal Justice Statistics, Washington, Department of Justice, L.E.A.A., N.C.U.I.S.S.

HINDELANG, J.J., T. HIRSCHI and J.G. WEIS (1979) : * Correlates of Delinquency : The Illusion of Discrepancy Between Self-Report and Official Measures ", American Sociological Review, vol. 44 (December), p. 995-1014.

HINDELANG, M., M. GOTTFREDSON and J. GAROFALO (1978) : Victims of Personal Crime : An Empirical Foundation for a Theory of Personal Victimization, Cambridge (Mass.), Ballinger Pub. Co.

HIRSCHI, T. (1969) : Causes of Delinquency, Berkeley, California, University of California Press.

HOOD, R. et R. SPARKS (1972) : la Delinquance, Paris, Hachette, L'Univers des connaissances.

H.U.D. (1979) : Voir U.S. Department of Housing and Urban Development.

LEBLANC, M. et M. FRECHETTE (1979) : La délinquance caché à l'adolescence s, Université de Montréal, Groupe de recherche sur l'inadaptation juvénile.

LEBLANC, M., L. BIRON, G. CÓTÉ et L. PRONOVOST (1979) : * La délinquance juvénile : son développement en regard de développement psycho-social durant l'adolescence ", Montrél, G.R.I.J., Université de Montréal.

MATTHEWS, K.W. (1976) : * Third Year Evaluation of the Community Crime Prevention Program from 1973 to 1976 *, Seattle, Law and Justice Planning Office, Unpublished report.

MATZA, D. (1954) : Delinquency and Drift, New York, Wiley.

MAXHEW, P.W. (1979) : Defensible Space : The Current Status of a Crime Prevention Theory ", The Howard Journal, vol. XVIII, p. 150-159.

MERTON, R.K. (1957) : Social Theory and Social Structure : Toward the Codification of Theory and Research, Glencoe, Ill., Free Press.

NEWMAN, O. (1972) : Defensible Space, New York, MacMillan.

N.C.J.I.S.S. (1980) : Expenditure and Employment Data for the Criminal Justice System 1978 ,, Washington (D.C.), Department of Justice, L.E.A.A., National Criminal Justice Information and Statistics Service, GSS No. 94P, SD-EE No. 15a. 
N.C.J.I.S.S. (1979a) : « Prisoners in State and Federal Institutions on December 31, 1978 ,, Washington (D.C.), Department of Justice, L.E.A.A., National Criminal Justice Information and Statistics Service, SD-NPS-PSF-6A.

N.C.J.I.S.S. (1979b) : C Children in Custody : Advance Report on the 1977 Census of Public Juvenile Facilities 》, Washington (D.C.), Department of Justice, L.E.A.A., National Criminal Information and Statistics Service, No. SD-JD-5A.

N.C.J.I.S.S. (1979c) : « Census of Jails and Survey of Jail Inmates 1978 ,, Washington (D.C.), Department of Justice, L.E.A.A., National Criminal Justice Information and Statistics Service, No. SD-NPS-J-6P.

OOSTHOEK, A. (1978) : Utilisation des statistiques officielles sur la criminalité ", Ottawa, Solliciteur général du Canada, Division de la recherche.

PERSPECTIVES CANADA III (1980) : Statistique Canada, Ottawa, Ministère des Approvisionnements et Services.

PIERCE, G. and W. BOWERS (1979) : The Impact of the Bartley-Fox Gun Law on Crime in Massachusetts *, Boston, Northeastern University Center for Applied Social Research.

REPPETTO, T. (1974) : Residential Crime, Cambridge (Mass.), Ballinger.

SCARR, H. (1973) : Patterns of Burglary, Washington (D.C.), National Institute of Law Enforcement and Criminal Justice.

SILBERMAN, C.E. (1978) : Criminal Violence, Criminal Justice, Toronto, Random House.

SKOGAN, G. (1975) : Measurement Problems in Official and Survey Crime Rates ,, Journal of Criminal Justice, vol. 3, p. 17-31.

SOLICITOR GENERAL OF CANADA (1980) : : Greater Vancouver Victimization Survey : Selected Highlights », Ottawa, Research and Statistics Divisions.

SOLLICITEUR GÉNERAL DU CANADA (1979a): Quelques tendances de la justice pénale canadienne, Ottawa, Groupe de la recherche et de la statistique.

SOLLICITEUR GENERAL DU CANADA (1979b) : Quelques statistiques choisies sur les homicides et les autres crimes de violence au Canada, Ottawa, Division de la statistique.

SOLLICITEUR GÉNÉRAL DU CANADA (1977) : Recueil statistiquejustice pénale du Canada, Ottawa, Division des systèmes d'information et de la statistique.

SOLLICITEUR GÉNÉRAL DU CANADA (1976) : Statistiques relatives au contrôle des armes à feu, Ottawa, Direction de la recherche et du perfectionnement des méthodes.

STATISTIQUE CANADA (1964-1970) : Statistiques de la criminalite, Ottawa.

STATISTIQUE CANADA (1972-1978) : Statistiques de la criminalité et de l'application des règlements de la circulation, Ottawa.

STENNING, P. and S. MOYER (1979) : * Gun Ownership and Use in Canada : A Report of Survey Findings, 1976 , Ottawa, Solicitor General of Canada, Research Division.

SOURCEBOOK (1979) : Voir Gottfredson et al. (1978). 
U.S. DEPARTMENT OF HOUSING AND URBAN DEVELOPMENT AND THE U.S. DEPARTMENT OF LABOR (1979) : Urban Initiatives Anti-Crime Program, Washington, Information Kit.

U.S. DEPARTMENT OF JUSTICE (1979) : Criminal Victimization in the United States 1977, Washington, Government Printing Office.

WALLER, I. (1974) : Man Released From Prison, Toronto, University of Toronto Press.

WALLER, I. and N. OKIHIRO (1978) : Burglary : The Victim and the Public, Toronto, University of Toronto Press.

WALLER, I. and J. CHAN (1974) : ex Prison Use : A Canadian and International Comparison ${ }^{*}$, Criminal Law Quarterly, vol. 17, 1974-75, p. 47-71.

WALLER, I. (1979) : Les moyens pour réduire le cambriolage; les solutions face aux faits *, Document presenté au Troisième colloque international sur la victimologie, tenu en septembre 1979 à Meunster, Westphalie, Allemagne.

WEIS, K. and E. MILAKOVICH (1974) : Political Misuses of Crime Rates ", Society, 11, 27-33. 